\title{
ON THE SECOND GAUSSIAN MAP FOR CURVES ON A K3 SURFACE
}

\author{
ELISABETTA COLOMBO AND PAOLA FREDIANI
}

\begin{abstract}
By a theorem of Wahl, for canonically embedded curves which are hyperplane sections of K3 surfaces, the first Gaussian map is not surjective. In this paper we prove that if $C$ is a general hyperplane section of high genus ( $>$ 280) of a general polarized K3 surface, then the second Gaussian map of $C$ is surjective. The resulting bound for the genus $g$ of a general curve with surjective second Gaussian map is decreased to $g>152$.
\end{abstract}

\section{$\S 1$. Introduction}

The first Gaussian map, or Wahl map, for the canonical series has been extensively studied, and it has been shown that for a general curve of genus $\geq 10$, different from 11, it is surjective (see [4], [18]).

Wahl ([19], see also [20], [21]) has given a deformation theoretic interpretation of the first Gaussian map, showing that if a canonical curve can be extended in projective space as a hyperplane section of a surface which is not a cone, then the first Gaussian map is not surjective. In particular, in [19] it is proven that if a curve lies on a K3 surface, the first Gaussian map cannot be surjective (see also [3]). The obstruction to the surjectivity of the first Gaussian map for a curve in a K3 surface is given by the extension class of the cotangent sequence

$$
0 \rightarrow K_{C}^{-1} \rightarrow \Omega_{X \mid C}^{1} \rightarrow K_{C} \rightarrow 0,
$$

which is a nontrivial element in the kernel of the dual of the first Gaussian map (see [3]).

This paper is concerned with the second Gaussian map, $\gamma_{C}^{2}: I_{2}\left(K_{C}\right) \rightarrow$ $H^{0}\left(C, 4 K_{C}\right)$. In fact, the second Gaussian map also encodes some interesting geometry. Our geometrical motivation to study it comes from its relation with the curvature of the moduli space $M_{g}$ of curves of genus $g$ with the

Received May 13, 2009. Revised November 10, 2009. Accepted December 4, 2009.

2000 Mathematics Subject Classification. 14H10, 14 J28.

(C) 2010 by The Editorial Board of the Nagoya Mathematical Journal 
Siegel metric induced by the period map $j: M_{g} \rightarrow A_{g}$, which we analyzed in [9]. There the curvature was computed using a formula for the associated second fundamental form given in [11]. In particular, in [11] it is proven that the second fundamental form lifts the second Gaussian map as stated in an unpublished paper of Green and Griffiths (see [12]).

In $[9,(3.8)]$ we gave a formula for the holomorphic sectional curvature of $M_{g}$ along a Schiffer variation $\xi_{P}$, for $P$, a point on the curve $C$, in terms of the holomorphic sectional curvature of $A_{g}$ and the second Gaussian map. The relation of the second Gaussian map with curvature properties of $M_{g}$ in $A_{g}$ suggested that its rank could give information on the geometry of $M_{g}$ and of some sublocus of it.

Another interesting problem is to understand whether the surjectivity of the second Gaussian map provides an obstruction to embed a curve in a surface as a hyperplane section. In this paper we address this problem for curves in a K3 surface, and we deduce results for the general curve.

Using cohomological techniques in the study of $\gamma_{C}^{2}$ for a curve in a K3 surface $X$, it is natural to consider the "symmetric square" of the cotangent extension

$$
0 \rightarrow \Omega_{X \mid C}^{1} \otimes K_{C}^{-1} \rightarrow S^{2} \Omega_{X \mid C}^{1} \rightarrow K_{C}^{2} \rightarrow 0
$$

This does not give any obstruction to the surjectivity of $\gamma_{C}^{2}$ for the general curve in a general K3 surface, while it gives an obstruction if $C$ is any curve in an abelian surface (see [10]). In fact, in [10] it is shown that if $C$ is a curve in an abelian surface $X$, then the corank of $\gamma_{C}^{2}$ is at least 2 .

In this paper (Theorem 3.1) we prove surjectivity of the second Gaussian map for a general curve $C$ of high genus (for all $g>280$ ) on a general polarized K3 surface. This clearly implies surjectivity for the general curve in the moduli space of curves of any genus $g>280$. In corollary (3.5) we decrease the lower bound for the genus of the general curve with a surjective second Gaussian map until 152, using examples given in [8]. Note that, for dimensional reasons, surjectivity can be expected for a general curve of genus at least 18, so our bound is far from being optimal; nevertheless, it is the first known lower bound.

We recall that examples of curves whose second Gaussian map is surjective were already given in [8] (for curves lying on the product of two curves) and in [1] (for complete intersections). Note that, using complete intersections, it is not possible to deduce surjectivity for the general curve of any sufficiently high genus, because of restrictions on the genus. Moreover, the 
first of such examples has a much higher genus. The examples of curves in a product of two curves are used in this paper to decrease the bound. On the other hand, Theorem 3.1 shows that general curves on K3 surfaces of sufficiently high genus behave as general curves in the moduli space, with respect to the second Gaussian map.

To prove our theorem, we first show that sufficient conditions for the surjectivity of $\gamma_{C}^{2}$ for $C$, a curve in a K3 surface $X$, are given by the surjectivity of the second Gaussian map $\gamma_{\mathcal{O}_{X}(C)}^{2}: I_{2}\left(\mathcal{O}_{X}(C)\right) \rightarrow H^{0}\left(S^{2} \Omega_{X}^{1} \otimes \mathcal{O}_{X}(2 C)\right)$ and the vanishing of $H^{1}\left(S^{2} \Omega_{X}^{1} \otimes \mathcal{O}_{X}(C)\right)$. To prove surjectivity of $\gamma_{\mathcal{O}_{X}(C)}^{2}$, we adapt the ideas used in [6] to prove surjectivity of the first Gaussian map $\gamma_{\mathcal{O}_{X}(C)}^{1}$.

More precisely, observe that a sufficient condition for the surjectivity of $\gamma_{\mathcal{O}_{X}(C)}^{2}$ is the vanishing of $H^{1}\left(I_{\Delta_{X}}^{3} \otimes p^{*}\left(\mathcal{O}_{X}(C)\right) \otimes q^{*}\left(\mathcal{O}_{X}(C)\right)\right)$, where $\Delta_{X}$ is the diagonal in $X \times X$ and $p, q$ are the two projections to $X$. The idea is to consider the blowup $Y$ of $X \times X$ along the diagonal $\Delta_{X}$ and to use the Kawamata-Viehweg vanishing theorem (see [13], [17]) as follows. Let $E$ be the exceptional divisor; denote by $\pi: Y \rightarrow X \times X$ the natural morphism and set $f:=p \circ \pi, g:=q \circ \pi$. Then

$$
\begin{aligned}
& H^{1}\left(I_{\Delta_{X}}^{3} \otimes p^{*}\left(\mathcal{O}_{X}(C)\right) \otimes q^{*}\left(\mathcal{O}_{X}(C)\right)\right) \\
& \quad \cong H^{1}\left(Y, f^{*}\left(\mathcal{O}_{X}(C)\right) \otimes g^{*}\left(\mathcal{O}_{X}(C)\right)(-3 E)\right) \\
& \quad \cong H^{1}\left(Y, f^{*}\left(\mathcal{O}_{X}(C)\right) \otimes g^{*}\left(\mathcal{O}_{X}(C)\right) \otimes K_{Y}(-4 E)\right)
\end{aligned}
$$

since $K_{Y}=\mathcal{O}_{Y}(E)$. So by the Kawamata-Viehweg vanishing theorem, it suffices to prove that the line bundle $L:=f^{*}\left(\mathcal{O}_{X}(C)\right) \otimes g^{*}\left(\mathcal{O}_{X}(C)\right)(-4 E)$ is big and nef.

Now notice that if one decomposes $\mathcal{O}_{X}(C)$ as $\bigotimes_{i=1}^{4} A_{i}$, where $A_{i}$ are line bundles on $X$, then $L=\bigotimes_{i=1}^{4}\left(f^{*}\left(A_{i}\right) \otimes g^{*}\left(A_{i}\right)(-E)\right)$. To obtain that $L$ is big and nef, we place suitable conditions on the line bundles $A_{i}$, and we study the sublinear system of $\left|f^{*}\left(A_{i}\right) \otimes g^{*}\left(A_{i}\right)(-E)\right|$ given by $\mathbb{P}\left(\Lambda^{2}\left(H^{0}\left(A_{i}\right)\right)\right)$ (see Lemma 3.3).

The vanishing of $H^{1}\left(S^{2} \Omega_{X}^{1} \otimes \mathcal{O}_{X}(C)\right)$ relies on a similar argument, but it requires a more refined version of it. In fact, given a decomposition of $\mathcal{O}_{X}(C)$ as $\mathcal{O}_{X}(D) \otimes \mathcal{O}_{X}\left(D^{\prime}\right)$, we have

$$
H^{1}\left(S^{2} \Omega_{X}^{1} \otimes \mathcal{O}_{X}(C)\right)=H^{1}\left(X \times X, I_{\Delta_{X}}^{2} / I_{\Delta_{X}}^{3} \otimes p^{*}\left(\mathcal{O}_{X}(D)\right) \otimes q^{*}\left(\mathcal{O}_{X}\left(D^{\prime}\right)\right)\right)
$$


hence, its vanishing is implied by the vanishing of $H^{1}\left(X \times X, I_{\Delta_{X}}^{2} \otimes\right.$ $\left.p^{*}\left(\mathcal{O}_{X}(D)\right) \otimes q^{*}\left(\mathcal{O}_{X}\left(D^{\prime}\right)\right)\right)$ and of $H^{2}\left(X \times X, I_{\Delta_{X}}^{3} \otimes p^{*}\left(\mathcal{O}_{X}(D)\right) \otimes\right.$ $\left.q^{*}\left(\mathcal{O}_{X}\left(D^{\prime}\right)\right)\right)$. So, with the same argument as above, it suffices to show that $f^{*}\left(\mathcal{O}_{X}(D)\right) \otimes g^{*}\left(\mathcal{O}_{X}\left(D^{\prime}\right)\right)(-4 E)$ is big and nef. The strategy is now to choose $\mathcal{O}_{X}(D)=\bigotimes_{i=1}^{4} A_{i}$ and $D^{\prime}=D+B$ with $B$ nef and effective, so that we take $C \in|2 D+B|$.

The above decompositions are shown on concrete examples of K3 surfaces $X$ and of curves $C$ in $X$, which are explicitly constructed via their Picard lattices (see Proposition 3.4).

Finally, regardless of the examples that we give, note that the conditions of the line bundles $A_{i}$ as in Lemma 3.3 and the decomposition $\mathcal{O}_{X}(C)=$ $\mathcal{O}_{X}(2 D+B)$ force the genus of $C$ to be far from the optimal lower bound. Regardless, observe that the vanishing of $H^{1}\left(S^{2} \Omega_{X}^{1} \otimes \mathcal{O}_{X}(C)\right)$ itself already implies that the curve $C$ must be of genus at least 31 , as one can check by looking at the restriction of $\Omega_{X}^{1} \otimes \Omega_{X}^{1}(C)$ to $C$ and the induced cohomology exact sequence.

\section{§2. Preliminaries on Gaussian maps}

Let $Y$ be a smooth complex projective variety, and let $\Delta_{Y} \subset Y \times Y$ be the diagonal. Let $L$ and $M$ be line bundles on $Y$. For a nonnegative integer $k$, the kth Gaussian map associated to these data is the restriction to diagonal map

$$
\begin{aligned}
& \gamma_{L, M}^{k}: H^{0}\left(Y \times Y, I_{\Delta_{Y}}^{k} \otimes L \otimes M\right) \rightarrow H^{0}\left(Y, I_{\Delta_{Y} \mid \Delta_{Y}}^{k} \otimes L \otimes M\right) \\
& \quad \cong H^{0}\left(Y, S^{k} \Omega_{Y}^{1} \otimes L \otimes M\right) .
\end{aligned}
$$

Usually first Gaussian maps are simply referred to as Gaussian maps. The exact sequence

$$
0 \rightarrow I_{\Delta_{Y}}^{k+1} \rightarrow I_{\Delta_{Y}}^{k} \rightarrow S^{k} \Omega_{Y}^{1} \rightarrow 0
$$

(where $S^{k} \Omega_{Y}^{1}$ is identified to its image via the diagonal map), twisted by $L \otimes M$, shows that the domain of the $k$ th Gaussian map is the kernel of the previous one:

$$
\gamma_{L, M}^{k}: \operatorname{ker} \gamma_{L, M}^{k-1} \rightarrow H^{0}\left(S^{k} \Omega_{Y}^{1} \otimes L \otimes M\right)
$$


In our applications, we will exclusively deal with Gaussian maps of order 2, assuming also that the two line bundles $L$ and $M$ coincide. For the reader's convenience, we spell out these maps. The map $\gamma_{L}^{0}$ is the multiplication map of global sections

$$
H^{0}(Y, L) \otimes H^{0}(Y, L) \rightarrow H^{0}\left(Y, L^{2}\right)
$$

which obviously vanishes identically on $\wedge^{2} H^{0}(L)$. Consequently, $H^{0}(Y \times$ $\left.Y, I_{\Delta_{Y}} \otimes L \otimes L\right)$ decomposes as $\wedge^{2} H^{0}(L) \oplus I_{2}(L)$, where $I_{2}(L)$ is the kernel of $S^{2} H^{0}(Y, L) \rightarrow H^{0}\left(Y, L^{2}\right)$. Since $\gamma_{L}^{1}$ vanishes on symmetric tensors, one writes

$$
\gamma_{L}^{1}: \wedge^{2} H^{0}(L) \rightarrow H^{0}\left(\Omega_{Y}^{1} \otimes L^{2}\right)
$$

Again, $H^{0}\left(Y \times Y, I_{\Delta_{Y}}^{2} \otimes L \otimes L\right)$ decomposes as the sum of $I_{2}(L)$ and the kernel of (4). Since $\gamma_{L}^{2}$ vanishes identically on skew-symmetric tensors, one usually writes

$$
\gamma_{L}^{2}: I_{2}(L) \rightarrow H^{0}\left(S^{2} \Omega_{Y}^{1} \otimes L^{2}\right)
$$

The primary object of this paper is the second Gaussian map of the canonical line bundle $K_{C}$ on a curve $C$ :

$$
\gamma_{C}^{2}: I_{2}\left(K_{C}\right) \rightarrow H^{0}\left(K_{C}^{4}\right)
$$

In our situation, $Y$ will be either a K3 surface $X$ or a smooth irreducible projective curve $C$ on $X$.

\section{§3. Main theorem}

THEOREM 3.1. If $X$ is a general polarized $K 3$ surface of degree $2 g-2$ with $g>280$, and if $C$ is a general hyperplane section of $X$, then $\gamma_{C}^{2}$ is surjective. 
Let us explain the strategy of the proof of Theorem 3.1. We have the following commutative diagram:

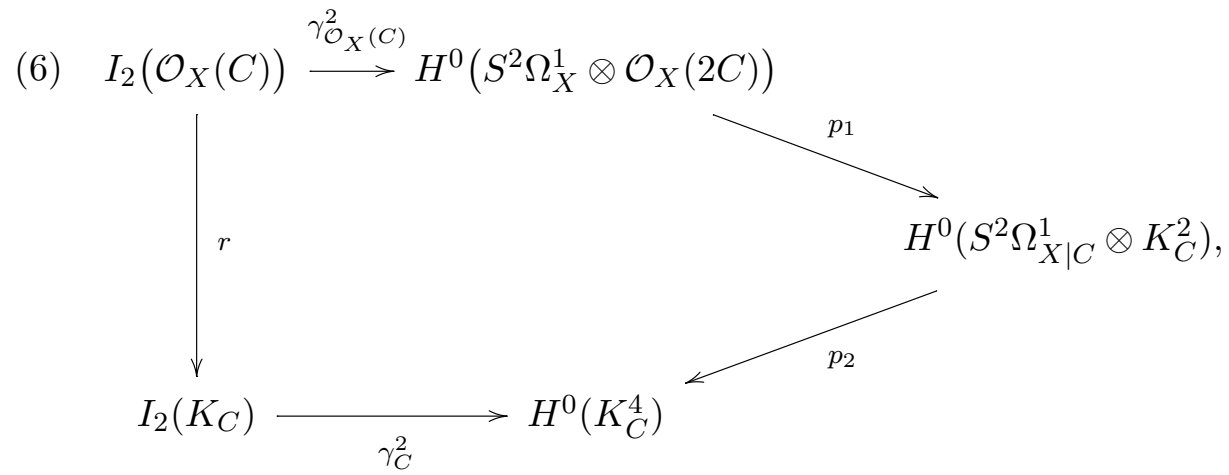

where $r$ and $p_{1}$ are restriction maps and $p_{2}$ comes from the conormal extension. More precisely, consider the exact sequence coming from the conormal extension

$$
0 \rightarrow \Omega_{X \mid C}^{1} \otimes K_{C} \rightarrow S^{2} \Omega_{X \mid C}^{1} \otimes K_{C}^{2} \rightarrow K_{C}^{4} \rightarrow 0
$$

then we have

$$
H^{0}\left(S^{2} \Omega_{X \mid C}^{1} \otimes K_{C}^{2}\right) \stackrel{p_{2}}{\rightarrow} H^{0}\left(K_{C}^{4}\right) \rightarrow H^{1}\left(\Omega_{X \mid C}^{1} \otimes K_{C}\right) \cong H^{0}\left(T_{X \mid C}\right)^{*},
$$

so $p_{2}$ is surjective by the following lemma.

LEMma 3.2. If $X$ is a general $K 3$ surface, and if $C$ is a general curve of genus at least 13 in the very ample linear system $\left|\mathcal{O}_{X}(C)\right|$, then $H^{0}\left(T_{X \mid C}\right)=0$.

Proof. By the exact sequence given by restriction of $T_{X}$ to $C, H^{0}\left(T_{X \mid C}\right)$ injects in $H^{1}\left(T_{X}(-C)\right)$, which vanishes by [7, Lemma 2.3].

The theorem will follow if we prove that the maps $\gamma_{\mathcal{O}_{X}(C)}^{2}$ and $p_{1}$ are also surjective. In fact, it suffices to exhibit examples of pairs $(X, C)$ where $X$ is a $\mathrm{K} 3$ and $C$ is a very ample curve in $X$ of any genus $g$ sufficiently high $(g \geq 281)$ for which $\gamma_{\mathcal{O}_{X}(C)}^{2}$ and $p_{1}$ are surjective. To do this, we follow the strategy used in [6] to study the first Wahl map (see also [5]). More precisely, from the exact sequence

$$
\begin{gathered}
0 \rightarrow I_{\Delta_{X}}^{3} \otimes p^{*}\left(\mathcal{O}_{X}(C)\right) \otimes q^{*}\left(\mathcal{O}_{X}(C)\right) \\
\rightarrow I_{\Delta_{X}}^{2} \otimes p^{*}\left(\mathcal{O}_{X}(C)\right) \otimes q^{*}\left(\mathcal{O}_{X}(C)\right) \\
\rightarrow I_{\Delta_{X}}^{2} / I_{\Delta_{X}}^{3} \otimes p^{*}\left(\mathcal{O}_{X}(C)\right) \otimes q^{*}\left(\mathcal{O}_{X}(C)\right) \rightarrow 0
\end{gathered}
$$


and taking global sections, we see that $\gamma_{\mathcal{O}_{X}(C)}^{2}$ is surjective if $H^{1}\left(I_{\Delta_{X}}^{3} \otimes\right.$ $\left.p^{*}\left(\mathcal{O}_{X}(C)\right) \otimes q^{*}\left(\mathcal{O}_{X}(C)\right)\right)=0$.

The idea used in [6] is to consider the blowup $Y$ of $X \times X$ along the diagonal $\Delta_{X}$ and to use the Kawamata-Viehweg vanishing theorem. Let $E$ be the exceptional divisor; denote by $\pi: Y \rightarrow X \times X$ the natural morphism and set $f:=p \circ \pi, g:=q \circ \pi$. Then

$$
\begin{aligned}
& H^{1}\left(I_{\Delta_{X}}^{3} \otimes p^{*}\left(\mathcal{O}_{X}(C)\right) \otimes q^{*}\left(\mathcal{O}_{X}(C)\right)\right) \\
& \quad \cong H^{1}\left(Y, f^{*}\left(\mathcal{O}_{X}(C)\right) \otimes g^{*}\left(\mathcal{O}_{X}(C)\right)(-3 E)\right) \\
& \cong H^{1}\left(Y, f^{*}\left(\mathcal{O}_{X}(C)\right) \otimes g^{*}\left(\mathcal{O}_{X}(C)\right) \otimes K_{Y}(-4 E)\right),
\end{aligned}
$$

since $K_{Y}=\mathcal{O}_{Y}(E)$. So, by the Kawamata-Viehweg vanishing theorem, it suffices to prove that $f^{*}\left(\mathcal{O}_{X}(C)\right) \otimes g^{*}\left(\mathcal{O}_{X}(C)\right)(-4 E)$ is big and nef.

Consider now the map

$$
p_{1}: H^{0}\left(S^{2} \Omega_{X}^{1} \otimes \mathcal{O}_{X}(2 C)\right) \rightarrow H^{0}\left(S^{2} \Omega_{X \mid C}^{1} \otimes K_{C}^{2}\right)
$$

Clearly $p_{1}$ is surjective if $H^{1}\left(S^{2} \Omega_{X}^{1} \otimes \mathcal{O}_{X}(C)\right)=0$.

Our strategy to prove the surjectivity of $p_{1}$ is to adapt the above idea for the vanishing of $H^{1}\left(Y, f^{*}\left(\mathcal{O}_{X}(C)\right) \otimes g^{*}\left(\mathcal{O}_{X}(C)\right) \otimes(-3 E)\right)$ in order to show that also $H^{1}\left(S^{2} \Omega_{X}^{1} \otimes \mathcal{O}_{X}(C)\right)$ vanishes. To this end, let $H$ be a very ample divisor, and assume that $C \in|2 H+B|$, where $B$ is nef and effective. Then

$$
\begin{aligned}
& H^{1}\left(S^{2} \Omega_{X}^{1} \otimes \mathcal{O}_{X}(C)\right) \\
& \quad \cong H^{1}\left(X, q_{*}\left(I_{\Delta_{X}}^{2} / I_{\Delta_{X}}^{3} \otimes p^{*}\left(\mathcal{O}_{X}(H)\right) \otimes q^{*}\left(\mathcal{O}_{X}(H+B)\right)\right)\right) \\
& \quad \cong H^{1}\left(X \times X, I_{\Delta_{X}}^{2} / I_{\Delta_{X}}^{3} \otimes p^{*}\left(\mathcal{O}_{X}(H)\right) \otimes q^{*}\left(\mathcal{O}_{X}(H+B)\right)\right)
\end{aligned}
$$

where the last isomorphism comes from Leray spectral sequence. So, by (7), to prove surjectivity of $p_{1}$ it suffices to show that $H^{1}\left(I_{\Delta_{X}}^{2} \otimes p^{*}\left(\mathcal{O}_{X}(H)\right) \otimes\right.$ $\left.q^{*}\left(\mathcal{O}_{X}(H+B)\right)\right)=0$ and that $H^{2}\left(I_{\Delta_{X}}^{3} \otimes p^{*}\left(\mathcal{O}_{X}(H)\right) \otimes q^{*}\left(\mathcal{O}_{X}(H+B)\right)\right)=0$. Using again the blowup $Y$, this is true if the line bundles $f^{*}\left(\mathcal{O}_{X}(H)\right) \otimes$ $g^{*}\left(\mathcal{O}_{X}(H+B)\right)(-4 E)$ and $f^{*}\left(\mathcal{O}_{X}(H)\right) \otimes g^{*}\left(\mathcal{O}_{X}(H+B)\right)(-3 E)$ are big and nef.

In conclusion, if we prove that $f^{*}\left(\mathcal{O}_{X}(H)\right) \otimes g^{*}\left(\mathcal{O}_{X}(H+B)\right)(-4 E)$ and $f^{*}\left(\mathcal{O}_{X}(H)\right) \otimes g^{*}\left(\mathcal{O}_{X}(H+B)\right)(-3 E)$ are big and nef, then $p_{1}$ is surjective. Moreover, also $f^{*}\left(\mathcal{O}_{X}(C)\right) \otimes g^{*}\left(\mathcal{O}_{X}(C)\right)(-4 E)$ is big and nef, and therefore $\gamma_{\mathcal{O}_{X}(C)}^{2}$ is surjective. 
Following [6] we will exhibit pairs $(X, C)$ as above (where $C \in \mid 2 H+$ $B \mid)$ for which $f^{*}\left(\mathcal{O}_{X}(H)\right) \otimes g^{*}\left(\mathcal{O}_{X}(H+B)\right)(-4 E)$ and $f^{*}\left(\mathcal{O}_{X}(H)\right) \otimes$ $g^{*}\left(\mathcal{O}_{X}(H+B)\right)(-3 E)$ are big and nef. First of all, observe that if there exist four line bundles $A_{i} i=1,2,3,4$ on $X$ such that $\mathcal{O}_{X}(H) \cong A_{1} \otimes A_{2} \otimes A_{3} \otimes A_{4}$, then we have

$$
\begin{aligned}
f^{*} & \left(\mathcal{O}_{X}(H)\right) \otimes g^{*}\left(\mathcal{O}_{X}(H+B)\right)(-4 E) \\
& \cong \bigotimes_{i=1,2,3,4}\left(f^{*}\left(A_{i}\right) \otimes g^{*}\left(A_{i}\right)(-E)\right) \otimes g^{*}\left(\mathcal{O}_{X}(B)\right),
\end{aligned}
$$

and

$$
\begin{aligned}
f^{*} & \left(\mathcal{O}_{X}(H)\right) \otimes g^{*}\left(\mathcal{O}_{X}(H+B)\right)(-3 E) \\
& \cong \bigotimes_{i=1,2,3}\left(f^{*}\left(A_{i}\right) \otimes g^{*}\left(A_{i}\right)(-E)\right) \otimes\left(f^{*}\left(A_{4}\right) \otimes g^{*}\left(A_{4} \otimes \mathcal{O}_{X}(B)\right)\right) .
\end{aligned}
$$

These are big and nef under the conditions given in the following lemma.

Lemma 3.3. Let $A_{1}, A_{2}, A_{3}, A_{4}$ be four base point free line bundles on a K3 surface $X$ with $A_{j}^{2} \geq 2, j=1,2,3,4$ and such that $A_{1}$ is very ample. Assume that either $A_{2}, A_{3}, A_{4}$ are very ample or they define (2:1) finite morphisms onto $\mathbb{P}^{2}$ and that if $A_{j}^{2}=2$, we have $\left(A_{1} \otimes A_{2} \otimes A_{3} \otimes A_{4}\right) \cdot A_{j} \geq 12$. Then

$$
\bigotimes_{i=1,2,3,4}\left(f^{*}\left(A_{i}\right) \otimes g^{*}\left(A_{i}\right)(-E)\right)
$$

is big and nef.

Proof. The proof is almost the same as the proof of [6, Lemma 2.2], but we reproduce it here for the reader's convenience.

If $A_{i}$ is very ample, the linear system $\left|f^{*}\left(A_{i}\right) \otimes g^{*}\left(A_{i}\right)(-E)\right|$ on $Y$ has a sublinear system defining the morphism $F: Y \rightarrow G r\left(1, \mathbb{P} H^{0}\left(A_{i}\right)^{*}\right)$, associating to $(x, y) \in Y$ the line between $\phi_{A_{i}}(x)$ and $\phi_{A_{i}}(y)$, composed with the Plücker embedding. Notice that if $(x, y) \in E$, we can think of $(x, y)$ as a pair where $x \in X, y \in \mathbb{P} T_{X, x}$; hence, $F(x, y)$ is the line generated by $\left(d \phi_{A_{i}}\right)_{x}(y)$. Therefore, $f^{*}\left(A_{i}\right) \otimes g^{*}\left(A_{i}\right)(-E)$ is nef, and it is also big, since the image of $X$ in $\mathbb{P} H^{0}\left(A_{i}\right)^{*}$ is not ruled.

Therefore, since $A_{1}$ is very ample, $\left(f^{*}\left(A_{1}\right) \otimes g^{*}\left(A_{1}\right)\right)(-E)$ is big; hence, $\bigotimes_{i=1,2,3,4}\left(f^{*}\left(A_{i}\right) \otimes g^{*}\left(A_{i}\right)(-E)\right)$ is big, and it can fail to be nef only on a curve $Z$ contained in the indeterminacy locus of the maps $Y \rightarrow \operatorname{Gr}(1$, 
$\left.\mathbb{P} H^{0}\left(A_{i}\right)^{*}\right), i=2,3,4$, if $A_{i}$ is not very ample. Notice that $Z$ is a curve contained in $\left\{(x, y) \in Y-E \mid \phi_{A_{i}}(x)=\phi_{A_{i}}(y)\right\} \cup\left\{(x, y) \in E \mid\left(d \phi_{A_{i}}\right)_{x}(y)=\right.$ $0\}$. Assume that $A_{i}$ is not very ample; hence, by assumption, it gives a $(2: 1)$ morphism to $\mathbb{P}^{2}$. If $Z \not \subset E$, let $\tau: X \times X \rightarrow X \times X$ be the involution $\tau(x, y)=(y, x)$; then we can assume that the image $\bar{Z}$ of $Z$ in $X \times X$ is such that $\tau(\bar{Z})=\bar{Z}$, because $\bigotimes_{i=1,2,3,4}\left(f^{*}\left(A_{i}\right) \otimes g^{*}\left(A_{i}\right)(-E)\right)$ is invariant under $\tau$. Then the first (or second) projection $Z^{\prime}$ of $\bar{Z}$ in $X$ is $\phi_{A_{i}}^{*}\left(Z_{1}\right)$ for some curve $Z_{1}$ in $\mathbb{P}^{2}$. If $L$ is a line in $\mathbb{P}^{2}$ and if $Z_{1} \sim m L$, we have

$$
\begin{aligned}
& \bigotimes_{i=1,2,3,4}\left(f^{*}\left(A_{i}\right) \otimes g^{*}\left(A_{i}\right)(-E)\right) \cdot Z \\
& \quad=2\left(A_{1} \otimes A_{2} \otimes A_{3} \otimes A_{4}\right) \cdot Z^{\prime}-4 E \cdot Z \\
& \quad=2\left(A_{1} \otimes A_{2} \otimes A_{3} \otimes A_{4}\right) \cdot m \phi_{A_{i}}^{*}(L)-4 E \cdot Z \\
& \quad=2 m\left(A_{1} \otimes A_{2} \otimes A_{3} \otimes A_{4}\right) \cdot A_{i}-4 E \cdot Z ;
\end{aligned}
$$

therefore, we are done if we show that $E \cdot Z=6 \mathrm{~m}$. Let $B$ be the ramification divisor of $\phi_{A_{i}}$; then $B$ is a smooth plane sextic, and $E \cdot Z=m B \cdot L=6 m$, if the intersection of $E$ and $Z$ is transverse. This can be checked directly as in $[6$, Lemma 2.2].

If $Z \subset E$, then it is the strict transform of the ramification divisor $R$ on $X$ of $\phi_{A_{i}}$; hence, $Z \cdot E=-c_{1}\left(\mathcal{O}_{\mathbb{P} T_{X}}(1)\right) \cdot Z=-\operatorname{deg} T_{R}=18$. Therefore,

$$
\bigotimes_{i=1,2,3,4}\left(f^{*}\left(A_{i}\right) \otimes g^{*}\left(A_{i}\right)(-E)\right) \cdot Z=6\left(A_{1} \otimes A_{2} \otimes A_{3} \otimes A_{4}\right) \cdot A_{i}-4 E \cdot Z \geq 0 .
$$

Let us now show the construction of the examples.

Proposition 3.4. There exist smooth K3 surfaces $X$ with Picard lattice $\Gamma=\mathbb{Z} D \oplus \mathbb{Z} L \oplus \mathbb{Z} R \oplus \mathbb{Z} S \oplus \mathbb{Z} T$ with intersection matrix $\operatorname{diag}(2 h,-2 k,-2 j$, $-2 l,-2 m)$ with $j, k, l, m \geq 2, h \geq k+1, j+1, l+1, m+1$ and $D$ very ample. Moreover, $D+L, D+R, D+S$, and $D+T$ are base point free, and they are either very ample or define $(2: 1)$ morphisms to $\mathbb{P}^{2}$.

Proof. Observe that the lattice $\Gamma$ is even, nondegenerate, and of signature $(1,4)$; hence, it occurs as the Neron-Severi group of some algebraic K3 surface (see [15, Corollary 2.9]). We will show that there does not exist a class $F \in \Gamma$ such that $F^{2}=-2, D \cdot F=0$. By well-known results on periods of K3 surfaces (see, e.g., [2]), this implies that there exists a $K 3$ surface with Picard lattice $\Gamma$ and such that $D$ is ample. 
Assume that $F=a D+b L+c R+d S+e T(a, b, c, d, e \in \mathbb{Z})$ is such that $F \cdot D=0$ and that $F^{2}=-2$. The first equality implies $a=0$, and the second one yields $1=k b^{2}+j c^{2}+l d^{2}+m e^{2}$, which is absurd, since $k, j, l, m \geq 2$. So $D$ is ample and $D^{2} \geq 4$; hence, $D$ is very ample provided that there does not exist an irreducible curve $F$ such that $F^{2}=0, F \cdot D=1,2$ (see [16], or [14, Theorem 5]). But this cannot happen, because if we write $F=a D+b L+c R+d S+e T, a, b, c, d, e \in \mathbb{Z}$, then $F \cdot D=2 h a \neq 1,2$ since $h \geq 2$. So $D$ is very ample. Note that $(D+L)^{2}=2 h-2 k \geq 2$, since $h \geq$ $k+1$.

First of all, we show that for any $(-2)$-curve $F, F \cdot(D+L)>0$; hence, $D+L$ is ample, and it is base point free, provided that there does not exist irreducible curves $F, G$ and an integer $a \geq 2$ such that $D+L \sim a F+G$, with $F^{2}=0, G^{2}=-2, F \cdot G=1$ (see [16], or [14, Theorem 5]). This clearly cannot happen, since the product of two classes is always even.

Set $F=a D+b L+c R+d S+e T, a, b, c, d, e \in \mathbb{Z}$ with $F^{2}=-2$. Since $D$ is ample, $D \cdot F=2 h a>0$; hence, $a>0 . F^{2}=-2$ yields $a^{2}=\left(k b^{2}+j c^{2}+\right.$ $\left.l d^{2}+m e^{2}-1\right) / h$.

If $(D+L)(a D+b L+c R+d S+e T)=2 a h-2 k b \leq 0$, we have $b k \geq a h>0$, so $b>0$ and $b^{2} k^{2} \geq h^{2} a^{2}=h\left(b^{2} k+c^{2} j+d^{2} l+e^{2} m-1\right)$. Thus we get

$$
b^{2}\left(k^{2}-h k\right)-h c^{2} j-h d^{2} l-h e^{2} m+h \geq 0,
$$

so if we set $h=k+1+t, t \geq 0$, we obtain

$$
\begin{aligned}
0 & \leq b^{2}\left(k^{2}-h k\right)-h c^{2} j-h d^{2} l-h e^{2} m+h \leq b^{2}\left(k^{2}-h k\right)+h \\
& =t\left(1-b^{2} k\right)+k\left(1-b^{2}\right)+1 \leq-t+1 ;
\end{aligned}
$$

hence, we must have either $t=0$ or $t=1$. But if $t=0$, then $h=k+1$, so

$$
\begin{aligned}
0 & \leq b^{2}\left(k^{2}-h k\right)-h c^{2} j-h d^{2} l-h e^{2} m+h \\
& =k\left(1-b^{2}\right)-(k+1)\left(c^{2} j+d^{2} l+e^{2} m\right)+1 \leq k\left(1-b^{2}\right)+1,
\end{aligned}
$$

so $b=1$, but then $-(k+1)\left(c^{2} j+d^{2} l+e^{2} m\right)+1 \geq 0$, which is absurd.

If $t=1$, then $h=k+2$, so we have

$$
0 \leq-2 b^{2} k+k+2-(k+2)\left(c^{2} j+d^{2} l+e^{2} m\right) \leq k\left(1-2 b^{2}\right)+2 ;
$$

thus we must have $b=1$, so $0 \leq-k+2-(k+2)\left(c^{2} j+d^{2} l+e^{2} m\right) \leq-(k+$ $2)\left(c^{2} j+d^{2} l+e^{2} m\right)$, which implies $c=d=e=0$. But then $a^{2}=(k-1) /(k+$ 
2 ), which is absurd. So $D+L$ is ample and base point free. If $(D+L)^{2} \geq 4$, a similar computation shows that there does not exist a curve $F$ such that $F^{2}=0$ and $F \cdot(D+L)=1,2$; therefore, $D+L$ is very ample.

If $(D+L)^{2}=2$, it clearly defines a $(2: 1)$ morphism to $\mathbb{P}^{2}$. The same holds for $D+R, D+S$, and $D+T$.

Proof of Theorem 3.1. Consider the K3 surfaces constructed in Proposition 3.4. Set $A_{1}=D$, and set $A_{i}, i=2,3,4$, equal to $D, D+L, D+R$, $D+S$, or $D+T$. By setting $H=A_{1}+A_{2}+A_{3}+A_{4}=4 D+a L+b R+c S+$ $d T$, with $a, b, c, d \geq 0, a+b+c+d \leq 3$, and $\tilde{H}=2 H+B$, where $B=n D+$ $m(D+L)+r(D+R)+s(D+S)+t(D+T)$, with $m, n, r, s, t \geq 0$, Lemma 3.3 applies. In fact, it suffices to check that $H \cdot(D+L) \geq 12, H \cdot(D+R) \geq 12$, and $H \cdot(D+S) \geq 12, H \cdot(D+T) \geq 12$, which is true for our choices of $H$. Hence, $\bigotimes_{i=1,2,3,4}\left(f^{*}\left(A_{i}\right) \otimes g^{*}\left(A_{i}\right)(-E) \otimes g^{*}\left(\mathcal{O}_{X}(B)\right)\right)$ is big and nef, and $\left(\otimes_{i=1,2,3}\left(f^{*}\left(A_{i}\right) \otimes g^{*}\left(A_{i}\right)(-E)\right)\right) \otimes\left(f^{*}\left(A_{4}\right) \otimes g^{*}\left(A_{4}\right) \otimes\left(\mathcal{O}_{X}(B)\right)\right)$ is also big and nef. If we take $C \in|\tilde{H}|$, we also have $H^{1}\left(S^{2} \Omega_{X}^{1} \otimes \mathcal{O}_{X}(C)\right)=0$; thus $\gamma_{C}^{2}$ is surjective.

Now we have to check that, with our choices of a nondivisible $\tilde{H}$, we obtain all the genera $g(C)=1+(1 / 2) \tilde{H}^{2} \geq 281$ for curves $C \in|\tilde{H}|$. To this end, it suffices to take $\tilde{H}=a D+s L+t R+v S+r T$, where $s, t, v, r \geq 0$, relatively prime, $s+t+v+r \leq a-2,9 \leq a \leq 14$, and, if $a=9$, at most one among $s, t, v, r$ is odd; if $a=10$, at most two among $s, t, v, r$ are odd; and if $a=11$, at most three among $s, t, v, r$ are odd.

In particular, let us start with $\tilde{H}=9 D+6 L+R$; for $C \in|\tilde{H}|$, we have

$$
g(C)=1+\frac{1}{2} \tilde{H}^{2}=1+81 h-36 k-j
$$

Let us set $n=k-2, m=j-2$, and $h=n+3+t$, where $n, m, t \geq 0$; then we have $h \geq m+3$, so $t \geq m-n, g(C)=1+81(n+3+t)-36(n+2)-(m+2)$, and we have two cases.

(1) $\rho:=n-m \geq 0, t \geq 0$; then

$$
\begin{aligned}
g(C) & =1+81(m+\rho+3+t)-36(m+\rho+2)-(m+2) \\
& =170+45 \rho+44 m+81 t
\end{aligned}
$$

with $t, m, \rho \in \mathbb{Z}, t, m, \rho \geq 0$. 
(2) $\alpha:=m-n \geq 0, t=\alpha+\beta$, with $\beta \geq 0$; then

$$
\begin{aligned}
g(C) & =1+81(n+3+\alpha+\beta)-36(n+2)-(n+2+\alpha) \\
& =170+44 n+80 \alpha+81 \beta,
\end{aligned}
$$

with $n, \alpha, \beta \in \mathbb{Z}, n, \alpha, \beta \geq 0$.

Since 44 and 45 are relatively prime, it is clear that with $g(C)=170+$ $45 \rho+44 m+81 t$ one gets all sufficiently high genera. Using (1) and (2), one can simply check that $g(C)$ runs through all the integers greater than 620 and that with the other choices of $\tilde{H}$ one gets all genera $g$ greater than 280 except for $g=321$.

For $g=321$, we consider the K3 surface constructed in [6, Proposition 3.2] with Picard lattice given by $\Gamma=\mathbb{Z} D \oplus \mathbb{Z} L$ with $D^{2}=4, L^{2}=2, D \cdot L=7$. In [6] it is proven that $D$ is very ample and that $L$ defines a $2: 1$ finite morphism onto $\mathbb{P}^{2}$. So if we set $A_{i}=D, i=1,2,3,4, H=4 D, B=3 D+L$, and $\tilde{H}=2 H+B=11 D+L$, since $H \cdot L=4 D \cdot L=28$, Lemma 3.3 applies. Hence, $\bigotimes_{i=1,2,3,4}\left(f^{*}\left(A_{i}\right) \otimes g^{*}\left(A_{i}\right)(-E) \otimes g^{*}\left(\mathcal{O}_{X}(B)\right)\right)$ is big and nef, and $\left(\otimes_{i=1,2,3}\left(f^{*}\left(A_{i}\right) \otimes g^{*}\left(A_{i}\right)(-E)\right)\right) \otimes\left(f^{*}\left(A_{4}\right) \otimes g^{*}\left(A_{4}\right) \otimes\left(\mathcal{O}_{X}(B)\right)\right)$ is also big and nef. So, as above, if we take $C \in|\tilde{H}|$, we also have $H^{1}\left(S^{2} \Omega_{X}^{1} \otimes\right.$ $\left.\mathcal{O}_{X}(C)\right)=0$; thus $\gamma_{C}^{2}$ is surjective. Now it suffices to check that $C$ has genus $g=1+(1 / 2) \tilde{H}^{2}=321$.

Corollary 3.5. For the general curve of genus greater than 152, the second Gaussian map $\gamma_{C}^{2}$ is surjective.

Proof. By Theorem 3.1 and semicontinuity of the corank of $\gamma_{C}^{2}$, for a general curve of genus greater than $280 \gamma_{C}^{2}$ is surjective. Surjectivity for the general curve of genus $153 \leq g \leq 280$ can be proved by exhibiting examples of curves of genus $g$ with a surjective second Gaussian map, which are either hyperplane sections of a polarized K3 surface, as in the proof of Theorem 3.1, or are contained in the product of two curves as in [8, Theorem 3.1].

More precisely, let $C_{1}, C_{2}$ be two smooth curves of respective genera $g_{1}$, $g_{2}$; choose divisors $D_{i}$ on $C_{i}$ of degree $d_{i}, i=1,2$. Set $X=C_{1} \times C_{2}$, and let $C \in\left|p_{1}{ }^{*}\left(D_{1}\right) \otimes p_{2}{ }^{*}\left(D_{2}\right)\right|$ be a smooth curve, where $p_{i}$ is the projection from $C_{1} \times C_{2}$ on $C_{i}$; then $g(C)=1+\left(g_{2}-1\right) d_{1}+\left(g_{1}-1\right) d_{2}+d_{1} d_{2}$.

In [8] we proved that if $g_{1}, g_{2} \geq 2, d_{i} \geq 2 g_{i}+5, i=1,2$, or if $g_{1} \geq 2, g_{2}=1$, $d_{1} \geq 2 g_{1}+5, d_{2} \geq 7$, or if $g_{2}=0, d_{2} \geq 7, d_{2}\left(g_{1}-1\right)>2 d_{1} \geq 4 g_{1}+10$, then $\gamma_{C}^{2}$ is surjective. 
Then one has to check directly that these values of $g(C)$ cover all the remaining integers between 153 and 280 .

\section{REFERENCES}

[1] E. Ballico and C. Fontanari, On the surjectivity of higher Gaussian maps for complete intersection curves, Ricerche Mat. 53 (2004), 79-85.

[2] A. Beauville, Preliminaires sur les periodes des surfaces K3, Asterisque 126 (1985), 91-97.

[3] A. Beauville and J.-Y. Merindol, Sections hyperplanes des surfaces K3, Duke Math. J. 55 (1987), 873-878.

[4] C. Ciliberto, J. Harris, and R. Miranda, On the surjectivity of the Wahl map, Duke Math. J. 57 (1988), 829-858.

[5] C. Ciliberto, A. F. Lopez, and R. Miranda, Projective degenerations of K3 surfaces, Gaussian maps, and Fano threefolds, Invent. Math. 114 (1993), 641-667.

[6] C. Ciliberto, A. F. Lopez, and R. Miranda, "On the corank of Gaussian maps for general embedded K3 surfaces" in Proceedings of the Hirzebruch 65 Conference on Algebraic Geometry (Ramat Gan, 1993), Israel Math. Conf. Proc. 9, Bar-Ilan University, Ramat Gan, 1996, 141-157.

[7] C. Ciliberto, A. F. Lopez, and R. Miranda, Classification of varieties with canonical curve section via Gaussian maps on canonical curves, Amer. J. Math. 120 (1998), $1-21$.

[8] E. Colombo and P. Frediani, Some results on the second Gaussian map for curves, Michigan Math. J. 58 (2009), 745-758.

[9] E. Colombo and P. Frediani, Siegel metric and curvature of the moduli space of curves, Trans. Am. Math. Soc. 362 (2010), no. 3, 1231-1246.

[10] E. Colombo, P. Frediani, and G. Pareschi, Hyperplane sections of abelian surfaces, preprint, to appear in J. Algebraic Geom., arXiv:math/0903.2781

[11] E. Colombo, G. P. Pirola, and A. Tortora, Hodge-Gaussian maps, Ann. Sc. Norm. Super. Pisa Cl. Sci. (4) 30 (2001), 125-146.

[12] M. L. Green, "Infinitesimal methods in Hodge theory" in Algebraic Cycles and Hodge Theory, Torino 1993, Lect. Notes Math. 1594, Springer, Berlin, 1994, 1-92.

[13] Y. Kawamata, A generalization of Kodaira-Ramanujam's vanishing theorem, Math. Ann. 261 (1982), 43-46.

[14] S. Mori, On degrees and genera of curves on smooth quartic surfaces in $P^{3}$, Nagoya Math. J. 96 (1984), 127-132.

[15] D. R. Morrison, On K3 surfaces with large Picard number, Invent. Math. 75 (1984), 105-121.

[16] B. Saint-Donat, Projective models of K3 surfaces, Amer. J. Math. 96 (1974), 602639.

[17] E. Viehweg, Vanishing theorems, J. Reine Angew. Math. 335 (1982), 1-8.

[18] C. Voisin, Sur l'application de Wahl des courbes satisfaisant la condition de BrillNoether-Petri, Acta Math. 168 (1992), 249-272.

[19] J. Wahl, The Jacobian algebra of a graded Gorenstein singularity, Duke Math. J. 55 (1987), 843-871.

[20] J. Wahl, Gaussian maps on algebraic curves, J. Differential Geom. 32 (1990), 77-98. 
[21] J. Wahl, "Introduction to Gaussian maps on an algebraic curve" in Complex Projective Geometry (Trieste, 1989/Bergen, 1989), London Math. Soc. Lect. Note Ser. 179, Cambridge University Press, Cambridge, 1992, 304-323.

Elisabetta Colombo

Dipartimento di Matematica

Università di Milano

I-20133, Milano

Italy

elisabetta.colombo@unimi.it

Paola Frediani

Dipartimento di Matematica

Università di Pavia

I-27100 Pavia

Italy

paola.frediani@unipv.it 